\title{
CONTIGUITY RELATIONS FOR GENERALIZED HYPERGEOMETRIC FUNCTIONS
}

\author{
ALAN ADOLPHSON AND BERNARD DWORK
}

ABstract. It is well known that the hypergeometric functions

$$
{ }_{2} F_{1}(\alpha \pm 1, \beta, \gamma ; t), \quad{ }_{2} F_{1}(\alpha, \beta \pm 1, \gamma ; t), \quad{ }_{2} F_{1}(\alpha, \beta, \gamma \pm 1 ; t),
$$

which are contiguous to ${ }_{2} F_{1}(\alpha, \beta, \gamma ; t)$, can be expressed in terms of

$$
{ }_{2} F_{1}(\alpha, \beta, \gamma ; t) \text { and }{ }_{2} F_{1}^{\prime}(\alpha, \beta, \gamma ; t) .
$$

We explain how to derive analogous formulas for generalized hypergeometric functions. Our main point is that such relations can be deduced from the geometry of the cone associated in a recent paper by B. Dwork and F. Loeser to a generalized hypergeometric series.

\section{INTRODUCTION}

Let $A=\left(A_{i j}\right)$ be an $(m \times n)$-matrix with entries in $\mathbb{Z}$. For $i=1, \ldots, m$, let $\ell_{i}$ be the linear form defined by the $i$ th row of $A$ :

$$
\ell_{i}\left(s_{1}, \ldots, s_{n}\right)=\sum_{j=1}^{n} A_{i j} s_{j} .
$$

Let $a=\left(a_{1}, \ldots, a_{m}\right) \in \mathbb{C}^{m}$. We suppose $a$ satisfies the condition:

$$
\text { If } a_{i} \in \mathbb{N}^{\times} \text {, then } A_{i j} \in \mathbb{N} \text { for } j=1, \ldots, n \text {, }
$$

where $\mathbb{N}$ denotes the nonnegative integers and $\mathbb{N}^{\times}$denotes the positive integers. We may then define the generalized hypergeometric series

$$
Y(a ; t)=\sum_{s \in \mathbb{N}^{n}} t_{1}^{s_{1}} \cdots t_{n}^{s_{n}} \frac{(-1)^{s_{1}+\cdots+s_{n}}}{s_{1} ! \cdots s_{n} !} \prod_{i=1}^{m}\left(a_{i}\right)_{\ell_{i}(s)},
$$

where as usual for $\rho \in \mathbb{Z},(z)_{\rho}=\Gamma(z+\rho) / \Gamma(z)$.

Let $\epsilon_{i}$ be the unit vector in the $i$ th coordinate direction in $\mathbb{C}^{m}$. It is easy to verify that if $a, a+\epsilon_{i}$ satisfy (1.1), then

$$
a_{i} Y\left(a+\epsilon_{i} ; t\right)=\left(a_{i}+\ell_{i}(\delta)\right) Y(a ; t),
$$

Received by the editors February 25, 1993; originally communicated to the Proceedings of the $A M S$ by Hal L. Smith.

1991 Mathematics Subject Classification. Primary 33C20, 33C65, 33C70.

The first author was supported in part by NSA Grant \#MDA904-91-H-0039.

(C)1995 American Mathematical Society $0002-9947 / 95 \$ 1.00+\$ .25$ per page 
where $\ell_{i}(\delta)=\sum_{j=1}^{n} A_{i j} \delta_{j}$ and $\delta_{j}=t_{j} \partial / \partial t_{j}$. The purpose of this note is to invert this relation. We solve the following problem.

Problem. Find $P_{i} \in \mathbb{Q}(a)\left[t, \partial / \partial t_{1}, \ldots, \partial / \partial t_{n}\right]$ such that for generic values of $a$,

$$
Y\left(a-\epsilon_{i} ; t\right)=P_{i}\left(a, t, \partial / \partial t_{1}, \ldots, \partial / \partial t_{n}\right) Y(a ; t) .
$$

We give an algorithm for constructing $P_{i}$ and show that the coefficients in $\mathbb{Q}(a)$ appearing in $P_{i}$ have denominators which are products of linear factors involving the faces of codimension one of the cone associated with $Y$ in earlier work $[1,2,3]$. We give estimates for the degree of $P_{i}$ as a polynomial in $t$ and we describe the set of $a \in \mathbb{C}^{m}$ for which (1.3) is valid. Under an additional condition, which is satisfied by all the classically studied hypergeometric series, we bound the order of $P_{i}$ as a partial differential operator.

This problem has a lengthy history. The function ${ }_{2} F_{1}$ had been treated by Gauss and Appell's $F_{1}$ had been treated by Lavasseur in his 1893 Paris thesis. Professor Kita has brought to our attention the recent works [5, 7]. The methods and scope of $[5,7]$ are quite different.

\section{EXPONENTIAL MODULES}

Let $A^{(j)}$ be the $j$ th column of the matrix $A$. We recall that, in earlier work, the polynomial

$$
-g(t, x)=x_{1}+\cdots+x_{m}+\sum_{j=1}^{n} t_{j} x^{A^{(j)}},
$$

where $x^{A^{(j)}}=x_{1}^{A_{1 j}} \cdots x_{m}^{A_{m j}}$, has been associated with $Y(a ; t)$. Let $\Omega=\mathbb{Q}(a)$, $R^{\prime}=\Omega(t)\left[x_{1}, x_{1}^{-1}, \ldots, x_{m}, x_{m}^{-1}\right], E_{i}=x_{i} \partial / \partial x_{i}$ for $i=1, \ldots, m$, and $g_{i}=E_{i}(g)$. Define operators on $R^{\prime}: D_{a, i, t}=E_{i}+a_{i}+g_{i}$ for $i=1, \ldots, m$, and $\sigma_{j}=\partial / \partial t_{j}-x^{A^{(j)}}\left(=\partial / \partial t_{j}+\partial g / \partial t_{j}\right)$ for $j=1, \ldots, n$. The $D_{a, i, t}$ and $\sigma_{j}$ commute with one another for all $i$ and $j$. We define

$$
\mathscr{W}_{a, t}^{\prime}=R^{\prime} / \sum_{i=1}^{m} D_{a, i, t} R^{\prime}
$$

which is viewed as an $\mathscr{R}_{1}$-module, where $\mathscr{R}_{1}$ is the noncommutative ring $\Omega(t)\left[\sigma_{1}, \ldots, \sigma_{n}\right]$.

We make the hypothesis

$$
a_{i} \notin \mathbb{N}^{\times} \text {for } i=1, \ldots, m .
$$

Let

$$
R^{*}=\left\{\sum_{u \in \mathbb{Z}^{m}} A_{u}(t) x^{-u} \mid A_{u}(t) \in \Omega[[t]]\right\}
$$

and let $\xi_{a, t}^{*} \in R^{*}$ be defined by

$$
\xi_{a, t}^{*}=\exp \left(-\sum_{j=1}^{n} t_{j} x^{A^{(j)}}\right) \cdot \sum_{u \in \mathbb{Z}^{m}} \frac{\prod_{i=1}^{m}\left(a_{i}\right)_{u_{i}}}{x^{u}} .
$$


By a direct calculation, for $u \in \mathbb{Z}^{m}$

$$
\left(\prod_{i=1}^{m}\left(a_{i}\right)_{u_{i}}\right) Y(a+u ; t)=\left\langle\xi_{a, t}^{*}, x^{u}\right\rangle,
$$

where for $\xi^{*} \in R^{*}, \xi \in R^{\prime},\left\langle\xi^{*}, \xi\right\rangle$ is defined to be the coefficient of $x^{0}$ in the product $\xi^{*} \xi$. In particular, taking $u=0$ gives

$$
Y(a ; t)=\left\langle\xi_{a, t}^{*}, 1\right\rangle .
$$

For any $\xi^{*} \in R^{*}, \xi \in R^{\prime}$, one checks easily that

$$
\frac{\partial}{\partial t_{j}}\left\langle\xi^{*}, \xi\right\rangle=\left\langle\sigma_{j}^{*}\left(\xi^{*}\right), \xi\right\rangle+\left\langle\xi^{*}, \sigma_{j}(\xi)\right\rangle,
$$

where $\sigma_{j}^{*}=\partial / \partial t_{j}+x^{A^{(j)}}$. From (2.2) it follows that $\sigma_{j}^{*}\left(\xi_{a, t}^{*}\right)=0$, hence

$$
\frac{\partial}{\partial t_{j}}\left\langle\xi_{a, t}^{*}, \xi\right\rangle=\left\langle\xi_{a, t}^{*}, \sigma_{j}(\xi)\right\rangle
$$

Applying this with $\xi=1$, we conclude that for $P \in \mathbb{Q}(a)\left[t, Z_{1}, \ldots, Z_{n}\right]$,

$$
\begin{aligned}
P\left(a, t, \partial / \partial t_{1}, \ldots, \partial / \partial t_{n}\right) Y(a ; t) & =P\left(a, t, \partial / \partial t_{1}, \ldots, \partial / \partial t_{n}\right)\left\langle\xi_{a, t}^{*}, 1\right\rangle \\
& =\left\langle\xi_{a, t}^{*}, P\left(a, t, \sigma_{1}, \ldots, \sigma_{n}\right) 1\right\rangle .
\end{aligned}
$$

Under the pairing $\langle$,$\rangle , the adjoint on R^{*}$ of the mapping $D_{a, i, t}$ on $R^{\prime}$ is the mapping $D_{a, i, t}^{*}=-E_{i}+a_{i}+g_{i}$. One checks that $D_{a, i, t}^{*}\left(\xi_{a, t}^{*}\right)=0$ for $i=1, \ldots, m$. It follows that $\xi_{a, t}^{*}$ annihilates $\sum_{i=1}^{m} D_{a, i, t} R^{\prime}$ under the pairing. Taking $u=-\epsilon_{i}$ in (2.3) and comparing with (2.5) reduces the problem stated in the introduction to the problem of finding $P_{i} \in \mathscr{R}_{1}$ such that

$$
\frac{a_{i}-1}{x_{i}} \equiv P_{i}\left(a, t, \sigma_{1}, \ldots, \sigma_{n}\right) 1\left(\bmod \sum_{i=1}^{m} D_{a, i, t} R^{\prime}\right) .
$$

One then has

$$
P_{i}\left(a, t, \partial / \partial t_{1}, \ldots, \partial / \partial t_{n}\right) Y(a ; t)=Y\left(a-\epsilon_{i} ; t\right) .
$$

Let $\ell_{i}(t \sigma)=\sum_{j=1}^{m} A_{i j} t_{j} \sigma_{j}$. One checks from the definitions that

$$
a_{i}+\ell_{i}(t \sigma)-\ell_{i}(\delta)=-E_{i}+x_{i}+D_{a, i, t},
$$

hence for $v \in \mathbb{Z}^{m}$,

$$
\left(a_{i}+\ell_{i}(t \sigma)+v_{i}\right) x^{v} \equiv x^{v+\epsilon_{i}} \quad\left(\bmod \sum_{i=1}^{m} D_{a, i, t} R^{\prime}\right) .
$$

One then proves by induction that for $r \in \mathbb{N}^{m}$,

$$
x^{r} \equiv \prod_{i=1}^{m}\left(a_{i}+\ell_{i}(t \sigma)\right)_{r_{i}} 1\left(\bmod \sum_{i=1}^{m} D_{a, i, t} R^{\prime}\right) .
$$


Let $\tilde{H}_{0}$ be the monoid generated by $\epsilon_{1}, \ldots, \epsilon_{m}, A^{(1)}, \ldots, A^{(n)}$. If $u \in \tilde{H}_{0}$, then $u=r+\sum_{j=1}^{n} s_{j} A^{(j)}$, where $r \in \mathbb{N}^{m},\left(s_{1}, \ldots, s_{n}\right) \in \mathbb{N}^{n}$. One has trivially

$$
\left(-\sigma_{1}\right)^{s_{1}} \cdots\left(-\sigma_{n}\right)^{s_{n}}\left(x^{r}\right)=x^{r+\sum_{j=1}^{n} s_{j} A^{(j)}}=x^{u} .
$$

Hence by (2.8),

$$
x^{u} \equiv\left(-\sigma_{1}\right)^{s_{1}} \cdots\left(-\sigma_{n}\right)^{s_{n}} \prod_{i=1}^{m}\left(a_{i}+\ell_{i}(t \sigma)\right)_{r_{i}} 1\left(\bmod \sum_{i=1}^{m} D_{a, i, t} R^{\prime}\right) .
$$

Thus to find $P_{i}$ satisfying (2.6), it suffices to find a formula of the type

$$
\frac{a_{i}-1}{x_{i}} \equiv \sum_{u \in \tilde{H}_{0}} c_{i, u} x^{u}\left(\bmod \sum_{i=1}^{m} D_{a, i, t} R^{\prime}\right),
$$

where the sum on the right-hand side is finite and each $c_{i, u}$ lies in $\mathbb{Q}(a)[t]$. For future use, we note that (2.9) combined with (2.5) gives for $u \in \tilde{H}_{0}$

$$
\left\langle\xi_{a, t}^{*}, x^{u}\right\rangle=(-1)^{s_{1}+\cdots+s_{n}} \prod_{j=1}^{n}\left(\frac{\partial}{\partial t_{j}}\right)^{s_{j}} \prod_{i=1}^{m}\left(a_{i}+\ell_{i}(\delta)\right)_{r_{i}} Y(a ; t) .
$$

\section{THE CONTIGUITY ALGORITHM}

Let $\mathscr{C}$ be the cone in $\mathbb{R}^{m}$ determined by the monomials of $g$ :

$$
\mathscr{C}=\left\{z \in \mathbb{R}^{m} \mid z=\sum_{i=1}^{m} r_{i} \epsilon_{i}+\sum_{j=1}^{n} s_{j} A^{(j)}, \text { all } r_{i}, s_{j} \in[0, \infty)\right\} .
$$

Let $\hat{H}_{0}=\mathscr{C} \cap \mathbb{Z}^{m}$. We introduce $\hat{H}_{0}$ because it can be characterized by a system of linear inequalities.

Lemma 3.1. There exists $w \in \tilde{H}_{0}$ such that $\hat{H}_{0}+w \subseteq \tilde{H}_{0}$. In particular, we may take $w=\sum_{i=1}^{m} T_{i} \epsilon_{i}$, where

$$
T_{i}=\sup \left(0,-1+\sum_{j=1}^{n} \sup \left(0,-A_{i j}\right)\right) .
$$

Remark. For classical hypergeometric functions the matrices $A$ are made explicit in the appendix of [2] and it is not hard to check that in all these cases we have $\tilde{H}_{0}=\hat{H}_{0}$, i.e., one may take $w=0$ in the classical examples.

Proof. If $v \in \hat{H}_{0}$ then $v=\sum_{i=1}^{m} r_{i} \epsilon_{i}+\sum_{j=1}^{n} s_{j} A^{(j)}$ where all $r_{i}$ and $s_{j}$ are nonnegative. Putting $r_{i}=\alpha_{i}+\alpha_{i}^{\prime}, \alpha_{i} \in \mathbb{N}, \alpha_{i}^{\prime} \in[0,1)$, and $s_{j}=\beta_{j}+\beta_{j}^{\prime}$, $\beta_{j} \in \mathbb{N}, \beta_{j}^{\prime} \in[0,1)$, we conclude that $v=u+\mu$, where $u \in \tilde{H}_{0}$ and $\mu=$ $\sum_{i=1}^{m} \alpha_{i}^{\prime} \epsilon_{i}+\sum_{j=1}^{n} \beta_{j}^{\prime} A^{(j)} \in \hat{H}_{0}$. Since $\mu$ lies in a bounded set, there are only a finite number of possibilities for $\mu$ and hence there exists $w \in \mathbb{N}^{m}$ such that $w+\mu \in \mathbb{N}^{m} \subseteq \tilde{H}_{0}$ for all $\mu$. This shows the existence of $w$. To check our particular choice for $w$ it is enough to check that for all $i, \alpha_{i}^{\prime}+\sum_{j=1}^{n} \beta_{j}^{\prime} A_{i j} \in \mathbb{Z}$ implies that $\alpha_{i}^{\prime}+\sum_{j=1}^{n} \beta_{j}^{\prime} A_{i j}+T_{i} \geq 0$. This follows from the fact that if 
$\inf _{j=1, \ldots, n}\left\{A_{i j}\right\}<0$, then

$$
\sum_{j=1}^{n} \beta_{j}^{\prime} A_{i j}>\sum_{j=1}^{n} \inf \left(0, A_{i j}\right)=-1-T_{i} .
$$

We now recall that the cone $\mathscr{C}$ may also be defined by linear inequalities. Let $\tau_{1}, \ldots, \tau_{\rho}$ be the hyperplanes through the faces of $\mathscr{C}$ of codimension one. Then for $k=1, \ldots, \rho, \tau_{k}$ is defined by a linear form

$$
f_{k}(u)=\sum_{i=1}^{m} B_{k i} u_{i}
$$

where the $B_{k i}$ are integers with greatest common divisor $1, f_{k}(u)=0$ is the equation of $\tau_{k}$, and $f_{k}(u) \geq 0$ for all $u \in \mathscr{C}$. Let us write

$$
\begin{aligned}
f_{k}\left(D_{a}\right) & =\sum_{i=1}^{m} B_{k i} D_{a, i, t} \\
f_{k}(g) & =\sum_{i=1}^{m} B_{k i} g_{i} \\
& =-\sum_{i=1}^{m} x_{i} f_{k}\left(\epsilon_{i}\right)-\sum_{j=1}^{n} t_{j} x^{A^{(j)}} f_{k}\left(A^{(j)}\right) \\
f_{k}(E) & =\sum_{i=1}^{m} B_{k i} E_{i} .
\end{aligned}
$$

The key point is that all monomials appearing in $f_{k}(g)$ have exponents lying in the region $f_{k}(u) \geq 1$.

Lemma 3.2. For each $v \in \mathbb{Z}^{m}$ there exists a representation

$$
x^{v} \equiv \sum_{u \in \tilde{H}_{0}} c_{v, u} x^{u} \quad\left(\bmod \sum_{i=1}^{m} D_{a, i, t} R^{\prime}\right),
$$

where the sum on the right-hand side is finite and each $c_{v, u} \in \mathbb{Q}(a)[t]$.

Proof. We use induction on $N_{v}=\sum_{k=1}^{\rho} \sup \left(0, f_{k}(w-v)\right)$. If $N_{v}=0$, then $f_{k}(v-w) \geq 0$ for all $k$ which shows that $v \in w+\hat{H}_{0} \subseteq \tilde{H}_{0}$ by Lemma 3.1. Thus we may assume $f_{k}(v-w)<0$ for some $k$. We compute

$$
f_{k}\left(D_{a}\right) x^{v}=\left(f_{k}(a)+f_{k}(v)\right) x^{v}+f_{k}(g) x^{v},
$$

and so

$$
x^{v} \equiv-\frac{1}{f_{k}(a)+f_{k}(v)} f_{k}(g) x^{v}\left(\bmod \sum_{i=1}^{m} D_{a, i, t} R^{\prime}\right) .
$$

We now apply the induction hypothesis to $f_{k}(g) x^{v}$, which is a $\mathbb{Z}[t]$-linear combination of terms $x^{v^{\prime}}$ such that $N_{v^{\prime}} \leq N_{v}-1$. 
Note that equation (3.3) remains valid under specialization of $a$ for all $a$ such that $f_{k}(a)+f_{k}(v) \neq 0$. As an immediate consequence of the proof, we have:

\section{Corollary 3.4.}

$$
\begin{gathered}
\operatorname{deg}_{t} c_{v, u} \leq N_{v}, \\
\left(\prod_{k=1}^{\rho}\left(f_{k}(a)+f_{k}(v)\right)_{\sup \left(0, f_{k}(w-v)\right)}\right) c_{v, u} \in \mathbb{Q}[a, t] .
\end{gathered}
$$

Specializing $v$ to $-\epsilon_{i}$ and combining Lemma 3.2 with (2.11), (2.3) and Corollary 3.4 gives:

Theorem 3.7. There exists $P_{i} \in \mathbb{Q}(a)\left[t, \partial / \partial t_{1}, \ldots, \partial / \partial t_{n}, \delta_{1}, \ldots, \delta_{n}\right]$ such that

$$
Y\left(a-\epsilon_{i} ; t\right)=P_{i}\left(a, t, \partial / \partial t_{1}, \ldots, \partial / \partial t_{n}, \delta_{1}, \ldots, \delta_{n}\right) Y(a ; t) .
$$

As polynomials in $t$, the coefficients of $P_{i}$ have degree bounded by

$$
N_{-\epsilon_{i}}=\sum_{k=1}^{\rho} \sup \left(0, f_{k}\left(w+\epsilon_{i}\right)\right)
$$

and $H_{i}(a) P_{i}$ has coefficients in $\mathbb{Q}[a, t]$, where

$$
H_{i}(a)=\prod_{i=1}^{\rho}\left(f_{k}(a)+f_{k}\left(-\epsilon_{i}\right)\right)_{\sup \left(0, f_{k}\left(w+\epsilon_{i}\right)\right)} .
$$

Thus this contiguity relation is valid provided $a_{j} \notin \mathbb{N}^{\times}$for $j=1, \ldots, m$ and $H_{i}(a) \neq 0$.

Of course, if one expresses $P_{i}$ as a polynomial in the $\partial / \partial t_{i}$ 's only (i.e., replace $\delta_{i}$ by $\left.t_{i} \partial / \partial t_{i}\right)$, then the degrees of its coefficients as polynomials in $t$ change. These new degrees can be bounded by the methods of the next section, under the additional assumption that $\hat{H}_{0}=\tilde{H}_{0}$.

We believe that this theorem gives the basic set of contiguity relations. We observe that other contiguity relations may be deduced from

$$
\left\langle\xi_{a, t}^{*}, D_{a, i, t} x^{v}\right\rangle=0
$$

for all $v \in \mathbb{Z}^{m}, i=1, \ldots, m$, together with either (2.11) for $v \in \tilde{H}_{0}$ or (2.3) for arbitrary $v \in \mathbb{Z}^{m}$.

\section{BOUNDING THE ORDER OF $P_{i}$}

To bound the order of $P_{i}$ as a differential operator, we introduce some auxiliary functions. For $u \in \tilde{H}_{0}$, put

$$
W(u)=\inf \left\{\sum_{i=1}^{m} r_{i}+\sum_{j=1}^{n} s_{j} \mid u=\sum_{i=1}^{m} r_{i} \epsilon_{i}+\sum_{j=1}^{n} s_{j} A^{(j)}, r_{i}, s_{j} \in \mathbb{N} \text { for all } i, j\right\} .
$$


From (2.11) we see that the differential operator on $Y(a ; t)$ that corresponds to $x^{u}$ (more precisely, that corresponds to a representation of $u$ minimizing $\left.\sum_{i=1}^{m} r_{i}+\sum_{j=1}^{n} s_{j}\right)$ has order $W(u)$. Thus the problem of bounding the order of the differential operator corresponding to $x^{v}, v \in \mathbb{Z}^{m}$, is reduced to the problem of bounding $W(u)$ as $u$ ranges over all terms with $c_{v, u} \neq 0$ on the right-hand side of Lemma 3.2. To accomplish this, we need to extend the definition of $W$ to all $u \in \mathbb{Z}^{m}$.

It is clear from (4.1) that if $u_{1}, u_{2} \in \tilde{H}_{0}$, then

$$
W\left(u_{1}+u_{2}\right) \leq W\left(u_{1}\right)+W\left(u_{2}\right) .
$$

Any $u \in \mathbb{Z}^{m}$ can be written $u=u_{1}-u_{2}$ with $u_{1}, u_{2} \in \tilde{H}_{0}$. If in addition $u \in \tilde{H}_{0}$, then (4.2) implies $W\left(u_{1}\right)-W\left(u_{2}\right) \leq W(u)$. Thus we may extend (4.1) by defining for $u \in \mathbb{Z}^{m}$

$$
W(u)=\sup \left\{W\left(u_{1}\right)-W\left(u_{2}\right) \mid u=u_{1}-u_{2}, u_{1}, u_{2} \in \tilde{H}_{0}\right\} .
$$

Remark. We shall establish later that, under the hypothesis $\hat{H}_{0}=\tilde{H}_{0}, W(u)<$ $\infty$ for all $u \in \mathbb{Z}^{m}$. This will show that our bound on the order of $P_{i}$ is nontrivial.

Lemma 4.4. If $u \in \mathbb{Z}^{m}, u^{\prime} \in \tilde{H}_{0}$, then

$$
W\left(u+u^{\prime}\right) \leq W(u)+W\left(u^{\prime}\right)
$$

Proof. Pick $u_{1}, u_{2} \in \tilde{H}_{0}$ such that $u+u^{\prime}=u_{1}-u_{2}$. By (4.2), $W\left(u^{\prime}+u_{2}\right) \leq$ $W\left(u^{\prime}\right)+W\left(u_{2}\right)$, hence

$$
W\left(u_{1}\right)-W\left(u_{2}\right) \leq W\left(u_{1}\right)+W\left(u^{\prime}\right)-W\left(u^{\prime}+u_{2}\right) .
$$

But $u_{1}-\left(u^{\prime}+u_{2}\right)=u$, so $W\left(u_{1}\right)-W\left(u^{\prime}+u_{2}\right) \leq W(u)$. This implies the lemma.

Proposition 4.5. For $v \in \mathbb{Z}^{m}$, the partial differential operator corresponding to $x^{v}$ under (2.11) and Lemma 3.2 has order $\leq W(v)+N_{v}$.

Proof. The proof is by induction on $N_{v}$. If $N_{v}=0$, then as noted in the proof of Lemma 3.2, $v \in \tilde{H}_{0}$. Suppose $N_{v}>0$. By Lemma 4.4, $f_{k}(g) x^{v}$ is a $\mathbb{Z}[t]$-linear combination of terms $x^{v^{\prime}}$ such that $W\left(v^{\prime}\right) \leq W(v)+1$. Since $N_{v^{\prime}} \leq N_{v}-1$, we are done by (3.3) and the induction hypothesis.

Corollary 4.6. The partial differential operator $P_{i}$ of Theorem 3.7 can be chosen to have order $\leq W\left(-\epsilon_{i}\right)+N_{-\epsilon_{i}}$.

We now show this bound is nontrivial when $\hat{H}_{0}=\tilde{H}_{0}$. We introduce a function $W^{\prime}$ on $\hat{H}_{0}$ defined by

$$
\begin{aligned}
& W^{\prime}(u)=\inf \left\{\sum_{i=1}^{m} r_{i}+\sum_{j=1}^{n} s_{j} \mid u=\sum_{i=1}^{m} r_{i} \epsilon_{i}+\sum_{j=1}^{n} s_{j} A^{(j)},\right. \\
& \left.\qquad r_{i}, s_{j} \in[0, \infty) \text { for all } i, j\right\} .
\end{aligned}
$$

Trivially, $W^{\prime}(u) \leq W(u)$ for all $u \in \tilde{H}_{0}$. The function $W^{\prime}$ has a geometric interpretation: $W^{\prime}(u)$ is the smallest nonnegative real number such that $u \in$ 
$W^{\prime}(u) \Delta$, where $\Delta$ is the convex hull of the points $\epsilon_{1}, \ldots, \epsilon_{m}, A^{(1)}, \ldots, A^{(n)}$ and the origin and $W^{\prime}(u) \Delta$ is the dilation of $\Delta$ by the factor $W^{\prime}(u)$. Let $\lambda_{1}, \ldots, \lambda_{p}$ be linear forms defining the codimension-one faces of $\Delta$ that do not contain the origin. We assume them to be normalized so that the corresponding codimension-one face lies in the hyperplane $\lambda_{i}(u)=1$ for $i=1, \ldots, p$. This determines the $\lambda_{i}$ 's uniquely. Then for $u \in \hat{H}_{0}$,

$$
W^{\prime}(u)=\sup \left\{\lambda_{i}(u) \mid i=1, \ldots, p\right\} .
$$

Lemma 4.9. Fix $u \in \hat{H}_{0}$. Then the set $\left\{W^{\prime}\left(u+u_{1}\right)-W^{\prime}\left(u_{1}\right) \mid u_{1} \in \hat{H}_{0}\right\}$ is bounded above and below.

Proof. From the definition of $W^{\prime}$ it is clear that

$$
W^{\prime}\left(u+u_{1}\right) \leq W^{\prime}(u)+W^{\prime}\left(u_{1}\right),
$$

hence the given set is bounded above by $W^{\prime}(u)$. By (4.8) we may choose $i_{1}, i_{2} \in\{1, \ldots, p\}$ such that

$$
W^{\prime}\left(u+u_{1}\right)=\lambda_{i_{1}}\left(u+u_{1}\right), \quad W^{\prime}\left(u_{1}\right)=\lambda_{i_{2}}\left(u_{1}\right) .
$$

Then $\left(\lambda_{i_{1}}-\lambda_{i_{2}}\right)\left(u_{1}\right) \leq 0$ but $\left(\lambda_{i_{1}}-\lambda_{i_{2}}\right)\left(u+u_{1}\right) \geq 0$, hence there exists $\alpha \in$ $[0,1]$ such that

$$
\left(\lambda_{i_{1}}-\lambda_{i_{2}}\right)\left(\alpha u+u_{1}\right)=0
$$

Then

$$
\begin{aligned}
W^{\prime}\left(u+u_{1}\right)-W^{\prime}\left(u_{1}\right) & =\lambda_{i_{1}}\left(u+u_{1}\right)-\lambda_{i_{2}}\left(u_{1}\right) \\
& =(1-\alpha) \lambda_{i_{1}}(u)+\alpha \lambda_{i_{2}}(u)
\end{aligned}
$$

by (4.10). This latter quantity is clearly bounded above and below independently of $u_{1}$.

Lemma 4.11. Suppose $\hat{H}_{0}=\tilde{H}_{0}$. There exists a positive constant $\kappa$ such that for all $u \in \tilde{H}_{0}$,

$$
W(u) \leq W^{\prime}(u)+\kappa .
$$

Proof. Let $u \in \tilde{H}_{0}$. Choose $r_{i}, s_{j} \in[0, \infty)$ such that

$$
u=\sum_{i=1}^{m} r_{i} \epsilon_{i}+\sum_{j=1}^{n} s_{j} A^{(j)} \quad \text { and } \quad W^{\prime}(u)=\sum_{i=1}^{m} r_{i}+\sum_{j=1}^{n} s_{j} .
$$

Put $[u]=\sum_{i=1}^{m}\left[r_{i}\right] \epsilon_{i}+\sum_{j=1}^{n}\left[s_{j}\right] A^{(j)} \in \tilde{H}_{0}$. Then $u-[u]=\mu \in \mathbb{Z}^{m} \cap \mathscr{C}=\tilde{H}_{0}$. Furthermore, $\mu$ lies in a bounded (hence finite) subset of $\tilde{H}_{0}$. Let $\kappa$ be the maximum value of $W$ on this finite subset. Now $u=[u]+\mu$, hence

$$
\begin{aligned}
W(u) & \leq \sum_{i=1}^{m}\left[r_{i}\right]+\sum_{j=1}^{n}\left[s_{j}\right]+\kappa \\
& \leq W^{\prime}(u)+\kappa .
\end{aligned}
$$

Proposition 4.12. Suppose $\hat{H}_{0}=\tilde{H}_{0}$. Then $W(u)<\infty$ for all $u \in \mathbb{Z}^{m}$. 
Proof. Write $u=u_{1}-u_{2}$ with $u_{1}, u_{2} \in \tilde{H}_{0}$. By Lemma 4.4, $W(u) \leq W\left(u_{1}\right)+$ $W\left(-u_{2}\right)$. Thus it suffices to show $W(-u)<\infty$ for all $u \in \tilde{H}_{0}$. So suppose $-u=u_{1}-u_{2}$ with $u, u_{1}, u_{2} \in \tilde{H}_{0}$. Then $u_{2}=u_{1}+u$, so

$$
\begin{aligned}
W\left(u_{1}\right)-W\left(u_{2}\right) & =W\left(u_{1}\right)-W\left(u_{1}+u\right) \\
& \leq W^{\prime}\left(u_{1}\right)+\kappa-W^{\prime}\left(u_{1}+u\right)
\end{aligned}
$$

by Lemma 4.11. By Lemma 4.9, this quantity is bounded above independently of $u_{1}$.

\section{EXAMPLES}

Consider the classical Gaussian hypergeometric function

$$
{ }_{2} F_{1}(\alpha, \beta, \gamma ; t)=\sum_{s=0}^{\infty} \frac{(\alpha)_{s}(\beta)_{s}}{(\gamma)_{s} s !} t^{s} .
$$

Using the relation $(\gamma)_{s}(1-\gamma)_{-s}=(-1)^{s}$ we have

$$
\begin{aligned}
{ }_{2} F_{1}(\alpha, \beta, \gamma ; t) & =\sum_{s=0}^{\infty}(\alpha)_{s}(\beta)_{s}(1-\gamma)_{-s} \frac{(-t)^{s}}{s !} \\
& =Y(\alpha, \beta, 1-\gamma ; t),
\end{aligned}
$$

so $a=\left(a_{1}, a_{2}, a_{3}\right)=(\alpha, \beta, 1-\gamma)$ and $\ell_{1}(s)=\ell_{2}(s)=s, \ell_{3}(s)=-s$. This corresponds to

$$
-g(t, x)=x_{1}+x_{2}+x_{3}+t \frac{x_{1} x_{2}}{x_{3}} \text {. }
$$

The codimension-one faces of the corresponding cone $\mathscr{C}$ are given by the forms $f_{1}(u)=u_{2}+u_{3}, f_{2}(u)=u_{1}+u_{3}, f_{3}(u)=u_{1}, f_{4}(u)=u_{2}$ and one checks that $\hat{H}_{0}=\tilde{H}_{0}$, hence $w=0$ in Lemma 3.1. One has $f_{1}\left(-\epsilon_{1}\right), f_{4}\left(-\epsilon_{1}\right) \geq 0$ but $f_{2}\left(-\epsilon_{1}\right)=f_{3}\left(-\epsilon_{1}\right)=-1$. Following the algorithm described in the proof of Lemma 3.2 by first applying $f_{2}\left(D_{a}\right)$ to $1 / x_{1}$ and then applying $f_{3}\left(D_{a}\right)$ to $x_{3} / x_{1}$ gives

$$
\frac{\alpha-1}{x_{1}} \equiv \frac{\alpha-1+x_{3}+t x_{2}}{\alpha-\gamma} \quad\left(\bmod \sum_{i=1}^{3} D_{a, i, t} R^{\prime}\right) .
$$

By (2.9),

$$
\begin{aligned}
& x_{3} \equiv(1-\gamma-t \sigma) 1\left(\bmod \sum_{i=1}^{3} D_{a, i, t} R^{\prime}\right), \\
& x_{2} \equiv(\beta+t \sigma) 1\left(\bmod \sum_{i=1}^{3} D_{a, i, t} R^{\prime}\right),
\end{aligned}
$$

so

$$
\frac{\alpha-1}{x_{1}} \equiv \frac{(\alpha-\gamma-t \sigma)+t(\beta+t \sigma)}{\alpha-\gamma} 1 \quad\left(\bmod \sum_{i=1}^{3} D_{a, i, t} R^{\prime}\right) .
$$


From (2.7) we get

$$
(\gamma-\alpha){ }_{2} F_{1}(\alpha-1, \beta, \gamma ; t)=\left(t(1-t) \frac{\partial}{\partial t}+(\gamma-\alpha-\beta t)\right){ }_{2} F_{1}(\alpha, \beta, \gamma ; t),
$$

a well-known classical formula (see [4, section 2.8 , equation (23)] or [6, Chapter VI, section 24]).

We give some details for the calculation of the contiguity relations for the Lauricella series $F_{A}$, which we write in the form

$$
Y(a ; t)=\sum_{s \in \mathbb{N}^{n}} c(s) \frac{t^{s}}{s_{1} ! \cdots s_{n} !},
$$

where

$$
c(s)=\left(a_{2 n+1}\right)_{s_{1}+\cdots+s_{n}} \frac{\prod_{i=1}^{n}\left(a_{i+n}\right)_{s_{i}}}{\prod_{i=1}^{n}\left(1-a_{i}\right)_{s_{i}}} .
$$

The associated polynomial [2] is

$$
-g=x_{1}+\cdots+x_{2 n+1}+\sum_{j=1}^{n} t_{j} x_{2 n+1} \frac{x_{n+j}}{x_{j}} .
$$

There are $2^{n}+2 n$ linear forms which define the associated cone:

$$
\begin{aligned}
f_{j}(u) & =u_{n+j} \quad(j=1, \ldots, n), \\
f_{n+j}(u) & =u_{n+j}+u_{j} \quad(j=1, \ldots, n),
\end{aligned}
$$

and for each subset $S$ of $\{1, \ldots, n\}$ the form

$$
f_{S}(u)=u_{2 n+1}+\sum_{j \in S} u_{j}
$$

Clearly these forms are nonnegative on the cone of $g$. We omit the proof that these forms define the cone.

We give some of the calculations for $Y\left(a-\epsilon_{1} ; t\right)$. By applying $f_{n+1}\left(D_{a}\right)$ to $1 / x_{1}$ we obtain

$$
\left(a_{n+1}+a_{1}-1\right) \frac{1}{x_{1}} \equiv\left(x_{1}+x_{n+1}\right) \frac{1}{x_{1}}=1+\frac{x_{n+1}}{x_{1}} .
$$

Letting $S=\{1\}$ and applying $f_{S}\left(D_{a}\right)$ to $x_{n+1} / x_{1}$ we obtain

$$
\left(a_{2 n+1}+a_{1}-1\right) \frac{x_{n+1}}{x_{1}} \equiv \frac{x_{n+1}}{x_{1}}\left(x_{1}+x_{2 n+1}+\sum_{j=2}^{n} t_{j} x_{2 n+1} \frac{x_{n+j}}{x_{j}}\right) .
$$

Letting $y_{l}=x_{2 n+1} x_{n+l} / x_{l}, l=1, \ldots, n$, this becomes

$$
\left(a_{2 n+1}+a_{1}-1\right) \frac{x_{n+1}}{x_{1}} \equiv x_{n+1}+y_{1}+\sum_{j=2}^{n} t_{j} y_{1} \frac{x_{n+j}}{x_{j}} .
$$


Thus we are reduced to the problem of reducing $y_{1} \cdots y_{l-1} x_{n+l} / x_{l}$ modulo $\sum_{i=1}^{2 n+1} D_{a, i, t} R^{\prime}$. Applying $f_{S}\left(D_{a}\right)$ with $S=\{1, \ldots, l\}$ we obtain

$$
\begin{aligned}
& \left(a_{2 n+1}+a_{1}+\cdots+a_{l}-1\right) y_{1} \cdots y_{l-1} \frac{x_{n+l}}{x_{l}} \\
& \quad \equiv\left(x_{1}+\cdots+x_{l}+x_{2 n+1}+\sum_{j=l+1}^{n} t_{j} y_{j}\right) y_{1} \cdots y_{l-1} \frac{x_{n+l}}{x_{l}} \\
& =y_{1} \cdots y_{l}+\sum_{i=1}^{l} y_{1} \cdots \hat{y}_{i} \cdots y_{l} x_{n+i}+\sum_{j=l+1}^{n} t_{j} y_{1} \cdots y_{l} \frac{x_{n+j}}{x_{j}} .
\end{aligned}
$$

By iteration we arrive at a representation of $1 / x_{1}$ as a polynomial in $x_{1}, \ldots$, $x_{2 n+1}, y_{1}, \ldots, y_{n}$ with coefficients in $\mathbb{Q}(a)[t]$. The number of steps is quite large since $f_{n+1}\left(-\epsilon_{1}\right)=-1$ and $f_{S}\left(-\epsilon_{1}\right)=-1$ for every subset $S$ of $\{1, \ldots, n\}$ that contains 1 (thus $N_{-\epsilon_{1}}=1+2^{n-1}$ ).

\section{REFERENCES}

1. B. Dwork, Cohomological interpretation of hypergeometric series, Rend. Sem. Math. Padova 90 (1993), 239-263.

2. B. Dwork and F. Loeser, Hypergeometric series, Japanese J. Math. 19 (1993), 81-129.

3. - Hypergeometric functions and series as periods of exponential modules, Perspectives in Mathematics, Academic Press (to appear).

4. A. Erdélyi, W. Magnus, F. Oberhettinger, and F. Tricomi, Higher transcendental functions, Vol. 1, McGraw-Hill, New York, 1953.

5. E. Horikawa, Transformations and contiguity relations for Gelfand's hypergeometric functions, preprint, Tokyo University.

6. E. G. C. Poole, Introduction to the theory of linear differential equations, Oxford Univ. Press, London and New York, 1936.

7. T. Sasaki, Contiguity relations of Aomoto-Gelfand hypergeometric functions and applications to Appell's system $F_{3}$ and Goursat's system ${ }_{3} F_{2}$, Siam J. Math. Anal. 22 (1991), 821-846. 74078

Department of Mathematics, Oklahoma State University, Stillwater, Oklahoma

E-mail address: adolphs@math.okstate.edu

Department of Mathematics, Princeton University, Princeton, New Jersey 08544

E-mail address: bmdwork@math.princeton.edu 